\title{
A New CBIR Approach for the Annotation of Medical Images
}

\author{
Mouhamed Gaith Ayadi \\ Department of computer \\ sciences \\ ISG university of Tunis \\ Tunisia
}

\author{
Riadh Bouslimi \\ Department of computer \\ sciences \\ ISG university of Tunis \\ Tunisia
}

\author{
Jalel Akaichi \\ Department of computer \\ sciences \\ ISG university of Tunis \\ Tunisia
}

\begin{abstract}
In the medical field, images, and especially digital images, are produced in ever increasing quantities and used for diagnostics and therapy. Imaging has occupied a huge role in the management of patients, whether hospitalized or not. This gave birth of the annotation of medical image process. The annotation is intended to image analysis and solve the problem of semantic gap. Physicians and radiologists feel better while using annotation techniques for faster making decision and giving solutions to patients in a faster and more accurate way. However, medical images annotation still a hard task specially the process based Content-based image retrieval (CBIR). Recently, advances in Content Based Image Retrieval prompted researchers towards new approaches in information retrieval for image databases. In medical applications it already met some degree of success in constrained problems. For this reason, we focus in this paper on presenting to provide an efficient semi-automatic tool which is used for efficient medical image retrieval from a huge content of medical image database and which is used for further medical diagnosis purposes for the new image annotation, because, efficient content-based image Retrieval in the medical domain is still a challenging problem. The goal of this work is to propose an approach able to compute similarity between a new medical image and old stored images. The annotator has to choose then one of the similar images and annotations related to the selected one are assigned to the new one. The idea is to apply an edge detector algorithm (Sobel algorithm) to the image and extract features from the filtered image by a color histogram. The edge to the image become likes Finger print to a human in our work. It is a search based edge. Edge representation of an image drastically reduces the amount of data to be processed, yet it retains important information about the shapes of objects in the scene. Edges in images constitute an important feature to represent their content and extraction features from filtered image improve searching of similar images, and keeping in the same time the properties of each image. The similarity measurement between images is developed based the Euclidean distance. The method can answer queries by example. The efficiency and performance of the presented method has been evaluated using the precision and the recall. The results of our experiments show high percentage of success, which is satisfactory.
\end{abstract}

\section{Keywords}

Annotation of medical images, Content Based Image Retrieval (CBIR), Euclidean distance, Color histogram, Semantic gap

\section{INTRODUCTION}

Advances in digital imaging technologies and the increasing prevalence of picture archival systems have led to an exponential growth in the number of images generated and stored in hospitals during recent years. Thus, automatic medical image annotation and categorization can be very useful for the purposes of image database management. In fact, the image is become probably one of the most important tools in medicine since it provides a method for diagnosis, monitoring drug treatment responses and disease management of patients with the advantage of being a very fast noninvasive procedure, having very few side effects and with an excellent cost-effect relationship. Doctors need to annotate these medical images and to analyze them to facilitate the access and taking care for the association of semantics to a medical image. We will work with annotation based CBIR.

Content based image retrieval (CBIR) is a technique in which images are indexed by extracting their low level features and image retrieval is only based upon these indexed image features. In an effective image retrieval system, the user poses a query and the system should find images that are somehow relevant to the query. Thus, a way of representing the query, a way of representing images, and a way of comparing a query and an image are needed. This kind of approach is known as querying by content.

CBIR has accepted different kinds of user queries for its implementation such as query by example, query by color, and query by relevance. When a query image is given, the image is processed to extract features in order to represent the image contents as numeric values. These values are called feature vectors which are used in the retrieval process. CBIR plays a pivotal role in medical image retrieval field such as CT scan images, MRI scan images, X-rays etc. Many systems already have been developed in this domain but each one has some difficulties in any way to provide efficient retrieval. So efficient content based image retrieval in medical domain is still a challenging problem.

The following [Figure1] depicts a Classical architecture of CBIR system. The goal of CBIR is to replicate this human ability of object recognition using a similar two step process: use of quantified measures from the image that are believed to represent color, shape, texture and interest points - the image descriptors - as an approach to human perception; use of machine learning techniques, to create a model for the data, or similarity measures, to interpret the image in order to establish the difference of two elements or groups of elements as an approach to human cognition. 


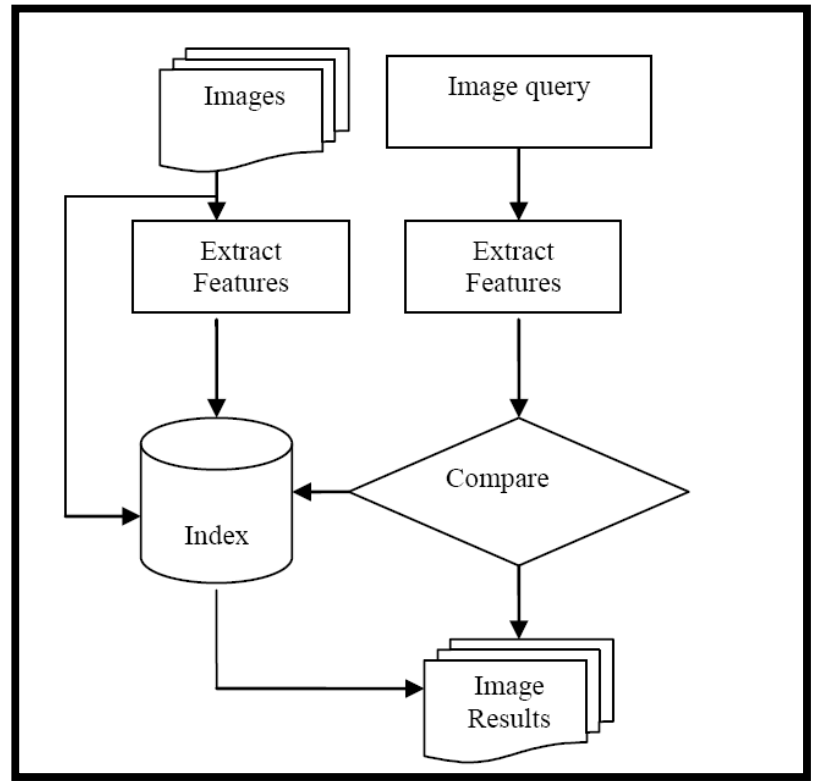

Figure 1. Architecture of CBIR system

\section{STATE OF THE ART}

In this section, we try to present all the concepts related to the annotation of medical images.

\subsection{Annotation of Medical Image}

The annotation of medical images is the task of assigning each image a set of tags or keywords. The annotation can be defined differently according to Barnard et al [1] "The purpose of the annotation is to assign keywords to specific regions of the image." According authors [2] "they are learned attributes to describe objects. "Wang et al [3] and Weston et al [4] order the most similar to ours must define a "description" of the image that could help visual search.

\subsection{Annotation types}

There are generally three types of image annotation: manual, semi-automatic and automatic. The manual annotation is usually performed by a librarian named iconographer. Its role is to assign each image categories and groups of words, often taken from a thesaurus to find images easily. However, when you have a large volume of images to annotate this work quickly becomes tedious, if not impossible; this is not the case for automatic annotation.

The automatic annotation: This is the task of associating a set of words in an image using a computer system without human intervention. The advantage of this system is that it gives the possibility to the user to pose queries in a high level language allowing him to express his information need easily. But sometimes it is inefficient and poorly annotated images. To make a compromise between these two tasks, the combination has become necessary. This is what is known as the "semi-automatic annotation."

\subsection{Medical Imaging Modalities}

The limitation of all the radiographic techniques is that their two-dimensional (2D) representation of the three-dimensional (3D) structures. The three imaging modalities used in the experimental setup were explained below. The scanning process is given below for all the three modalities.

- Computed Tomography: Computed tomography (CT) obtains a series of different angular X-ray projections that are processed by a computer to give a section of specified thickness. The CT image comprises a regular matrix of picture elements (pixel). Modern multiscale helical CT scanners can obtain images in seconds, and imaging of the whole body from the loop of the head to the thighs akes a single breadth hold of 20-30 s. The faster scan times allow dynamic imaging of arteries and veins at different times after the injection of intravenous contrast agents. Generally, studies were performed with patient spine and images were obtained in the transverse or axial plain. Modern CT scanners allow up to 25 degrees of gantry angulations, particularly which is valuable in spinal imaging. Occasionally, direct coronal images were obtained in the investigation of cranial and maxilla facial abnormalities; in these cares, the patient lies prone with the neck extended and the gantry appropriately angled.

- Magnetic Resonance Imaging: Magnetic resonance imaging (MRI) images can be obtained in any orthogonal or non-orthogonal plane. There is a wide range of pulse sequences, each of which provides a different image contrast. An intravenous injection of contrast medium (a gadolinium complex) may be given to enhance the visualization of tumors, inflammatory and vascular abnormalities.

- Ultrasound: Ultrasound (US) images do not depend on the use of electromagnetic wave format. It is the properties of high-frequency sound waves (longitudinal waves) and their interaction with biological tissues. Interpretation of the anatomy from static US images is more difficult than that from the other imaging modalities, because the technique is highly operator dependent and provides information on tissue structure and is different from the other imaging techniques. Tissue characterization is excellent in US. It can also detect lesions.

\subsection{Image Descriptors:}

\section{- The histogram}

The histogram [5] shows the proportion of pixels of each color in an image. On the question, the user has two options: either to specify the percentage of each desired color or to propose a model picture.

\section{- The texture descriptors}

The texture [6] is the difference between the pixels in an image. In fact, this kind of descriptor is used when it comes to measure the similarity between the regions of two images of the same color. The interrogation by the texture can be done in the same way as the color (selected examples of textures, image presentation models)

It is based on various methods such as: co-occurrence matrix, the Fourier transform of the Gabor filter, wavelet...

\section{- Shape descriptors}

This type allows you to compare two images with objects of the same shape [7]. It offers the user to formulate his query using either a model image or predefined shapes compared to all images stored in the database. The shape features are often extracted from the segmentation of images.

\section{- SIFT}

The descriptor SIFT (Scale Invariant Feature Transform) [5] describes the neighborhood of a point by building a histogram of gradient orientations. The gradient vector (direction and amplitude) is calculated for each pixel scale corresponding to the neighborhood. Directions are discretized to have only more than 8 directions. The neighborhood is divided into a grid.

\section{- SURF (speeded Up Robust Features)}


Also said robust feature is an accelerated algorithm for detecting characteristic presented by researchers at the ETH Zurich and the Katholieke Universiteit Leuven. Its main objective is to accelerate the various image processing. SURF [5] is partly inspired by the SIFT descriptor, it outperforms fast and is more robust for different image transformations.

\subsection{Image Retrieval Models}

According to [8], we identify three types of indexing models:

- the Boolean model

- the vector model

- And the probabilistic model.

For The Boolean model, the images are first characterized by a list of descriptors and the query is a logical formula that combines descriptors examples and logical operators (AND, OR, NOT). In response, the system is classified into two classes corresponding firstly to images that match the query and also to those who do not comply.

In the case of vector model, the query image and target image, that is to say, the images of the database are represented by a vector in a space attribute.

This vector corresponds to the concatenation of the basic weights of descriptors. A function of similarity between vectors is used to classify the images according to their relevance to the query.

For the probabilistic model, there assigning a relevance probability of the image in response to the request, each of the descriptors. Under the hypothesis of independence of the descriptors, it is possible to calculate the probability that the image meets the user's query as the product of the previous probabilities.

This type of model uses a strong user interaction through, for example, of relevance judgments issued by the user on the proposals made by the system results.

\subsection{CBIR Content-Based Image Retrieval}

In practice the conceptualization of a general thesaurus of medical terms consume many resources and demands extensive collaboration efforts where consensus is hard to reach. It is reasonable to use inductive approaches by starting with more specific standards and attempt generalization later. In the composite SNOMED-DICOM micro-glossary [9] such a strategy is used. Nevertheless, all standards presented are not ineffectual since they are used in several Picture Archive and Communications Systems (PACS). Facing the amount of images in a database, annotation by human hand can be a time consuming and cumbersome task where perception subjectivity can lead to unrecoverable errors. A study of medical images using DICOM headers revealed $15 \%$ of annotation errors from both human and machine origin [10]. The amount of different languages that can be used for annotation is extensive and may lead to translation/interpretation errors during a search statement or when databases are merged. It is convenient to be aware of the prospect of re-indexing images due to the presence of an event that changes the importance of a particular aspect, e.g., Forsyth's previously unknown famous person photos [11], or the need to link the content of the image to a new search statement possibility, e.g., Seloff's engineer search for a misaligned mounting bracket existent only in a annotated astronaut training image [12]. Another major obstacles for concept-based image retrieval systems are the existence of homographs and the fact that the search statement, or query, does not allow the user to switch and/or combine interaction paradigms [13] during text transactions. The ideal system would relieve the human factor from the annotation task, by doing it automatically, and allowing image retrieval by its content in its purest form, not only by text description. This is Content Based Image Retrieval (CBIR).

\subsection{Distances Measures}

To perform the actual medical image retrieval, authors [14] investigated a number of distance measure to discover which can give the most accurate and perceptually correct results.

- The Euclidean Distance: The direct Euclidean distance calculation between a database image $\mathrm{p}$ and query image $\mathrm{q}$ is given below. By using Eq. as follows Euclidean distance metric is made possible.

$$
\text { Euclidiean distance }=\sqrt{\sum\left(v_{p i-} v_{q i}\right)^{2}}
$$

- Manhattan Distance: The Manhattan distance function computes the distance from one data point to the other in a grid-like path. The Manhattan distance between two items is the sum of the differences of their corresponding points. Equation as follows shows the way to calculate Manhattan distance.

$$
\text { Manhattan distance }=\sum\left|v_{p i}-v_{q i}\right|
$$

- Mahalanobis Distance: The Mahalanobis distance is a special case of the quadratic-form distance matrix. The transform matrix is given by the covariance matrix obtained from the training set of feature vectors. Feature vectors were treated as random variables. By using Eq. as follows, we can calculate the Mahalanobis distance between query image and images in database.

Mahalanobis distance $=\left[\left(x_{q}-x_{d}\right) \sum\left(x_{q}-x_{d}\right)\right]^{1 / 2}$

- Canberra Distance: The Canberra distance is defined as follows:

$$
\text { Canberra distance }=\frac{\left|x_{i}^{k}-x_{j}^{k}\right|}{\left|x_{i}^{k}+x_{j}^{k}\right|}
$$

Where $x_{i}^{k}$ is the query image and $x_{j}^{k}$ is the database image which is shown in the Eq.

- Bray-Curtis: It is a statistic used to quantify the compositional dissimilarity between the two sets. The distance metric for Bray-Curtis is given in the Eq. as follows.

$$
\text { Bray }- \text { Curtis }=\sum \frac{\left(x_{i}-y_{i}\right)}{\left(x_{i}+y_{i}\right)}
$$

Where $\mathrm{i}=1$ to n. $x_{i}$ is the query image and $y_{i}$ is the image in the database.

- Squared Chord: The distance metric for squared chord can be calculated by using Eq. as follows.

$$
\text { Squared Chord }=\sum_{k}\left(\sqrt{p_{i k}}-\sqrt{p_{j k}}\right)^{2}
$$

Where $p_{i k}$ is the query image and $p_{j k}$ is the image in the database.

- Chi-Squared: One recommended dissimilarity coefficient in combination with compositional data is 
the chord distance. The chord distance between query image and database image is,

$$
\text { Chi-squared }={\sqrt{\sum_{k}\left(x_{i j}^{0.5}-x_{i k}^{0.5}\right)}}^{2}
$$

Where $\mathrm{k}=1$ to $\mathrm{m} . x_{i j}$ is the query image and $x_{i k}$ is the image in the database, which can be calculated using this Eq.

\subsection{Edge Detection Algorithms}

Edges form the outline of an object and when efficiently identified can locate all the objects in an image. Moreover, basic properties such as area, perimeter can be measured easily. The edge of a bone structure is one of the most meaningful parts used during extraction from background. The frequently used edge detectors are Sobel, Prewitt, Roberts, Canny and Laplacian of Guassian (LoG) algorithms. Edge detection algorithms are grouped into two categories, namely, Gradient operator and Laplacian operator [15]. Gradient operator detects edge pixel by obtaining the maximum and minimum value at first derivative level on the image. The classical gradient operators selected in this work are Sobel, prewitt, Robert, Canny [16] [17]. Laplacian Operator is a second order derivative, where the value of edge pixel at the first derivative is referred to as zero-crossing at second order derivative. The disadvantage of this operator is its sensitive feature towards noise effect. In solving this problem, Gaussian function is being applied on the image. This is terms are Laplacian of Gaussian (LoG). The operators are explained below.

- Sobel operator: Sobel edge detection is used in image processing techniques. The sobel kernals are more suitable to detect edges along the horizontal (180 degree) and vertical axis (90 degree) [18]. The sobel operator is based o convolving the image with a small, separable, and integer valued filter.

- Canny Edge Detector: Canny edge detection [19] is a multistage algorithm to detect a wide range of edge in images. It was presented in 1986 by Canny. The problem with this type of traditional edge detection approach is that a low threshold produces false edges, but a high threshold misses important edges.

- Prewitt edge detection: Prewitt operator edge detection masks [19] are the one of the oldest and best understood methods of detecting edges in images. The strength of the edge at given location is then the square rote of the sum of the squares of two derivatives.

- Robert edge detection: In Robert edge detection [19], the vertical and horizontal edges bring out individually and then put together for resulting edge detection. The two individual images $G_{x}$ and $G_{y}$ will be combined to get result. The Robert cross kernels are relatively small .Therefore they are highly susceptible to noise.

- Laplacian of Gaussian (LOG): This method of edge detection was invented by Marr and Hildreth in 1980.In this method, the Gaussian filtering is combined with Laplacian to break down the image where the intensity varies to detect the edges effectively. It finds the correct place of edges and testing wider area around the pixel .The disadvantage of LOG operator is that it cannot find orientation of edge because of laplacian filter [19].

\section{RELATED WORK}

CBIR in the medical field also presents a growing trend in publications [20]. Although the number of experimental algorithms comprehending specific problems and databases face a growth its reflection on the number of medical applications and frameworks is still very constrained. Only a few systems exist with relative success. The CervigramFinder system [21] was developed to study the uterine cervix cancer. It is a computer assisted framework where local features from a user-defined region in an image are computed and, using similarity measures, similar images are retrieved from a database. The Spine Pathology \& Image Retrieval System (SPIRS) [22] is a web-based hybrid retrieval system, working with both image visual features and text-based information. It allows the user to extract spine $\mathrm{x}$-rays images from a database by providing a sketch/image of the vertebral outline. The retrieval process is based in a active contours algorithm for shape discrimination. The Image Retrieval for Medical Applications (IRMA) system [23] is a generic web-based $\mathrm{x}$ ray retrieval system. It allows the user to extract images from a database given an x-ray image query. Local features and similarity measures are used to compute the nearest images. The SPIRS and IRMA systems were merged to form the SPIRS-IRMA system, with the functionalities of both. More recently a CBIR framework prototype was proposed for retrieval of images from a broader domain, including X-rays, CT and US [24]. In this system multiple features from the image, based in intensity, shape and texture, are extracted from a given query and used to retrieve similar images based on similarity measures. Reviews of CBIR for medical applications can be found in [25] and [26]. A review of 21 CBIR systems for Radiology can be found in [27]. The Image Map [28] is one of the existing medical image retrieval that considers how to handle multiple organs of interest. However, it works based on spatial similarity. Consequently, a problem caused by user is likely to occur and therefore, the retrieved image will represent an unexpected organ.

The Automatic Search and Selection Engine with Retrieval Tools (ASSERT) [29]. A physician-in-the-Loop content-based retrieval system for HRCT image databases which is implemented to show a human-in-the-loop approach in which the human delineates the pathology bearing regions(PBR) and a set of anatomical landmarks in the image when the image is entered into the database.

The MIMS [30] takes the complexity first when describing semantic content of images and second the graphical aspect of certain objects. This existence creates some critical problems of subjectivity, although such an approach is made as general as possible not to specific one. The WebMIRS [31] with this system, the user manipulates GUI tools to create a query. In response the system returns the values for given user query and displays the associated $\mathrm{x}$-ray images.

Medical applications are one of the priority areas where CBIR can meet more success outside the experimental sphere due to population aging in developed countries. Notwithstanding the progress already achieved in the few frameworks available here is still a lot of work to be done in order to develop a commercial system able to fulfill image retrieval/diagnosis comprehending a broader image domain.

\section{PROPOSED METHOD}

In this system, the input query image is given at runtime to get her edge by the application of an edge detector. Afterward, the process of features extraction will be done on filtered image. Edge detection is a fundamental tool, which is commonly used in many image processing applications to obtain information from medical images [19]. The separation of the image into object and background is a critical step in image interpretation. The processing of database images consist of three main stages namely: image edge detector, image feature extraction, and indexing that have been used in order to retrieve the similar images from the database. For retrieval 
process, Euclidean distance finding method has been used. The following processing steps have been applied in order to perform the retrieval of similar images from the database:

1. Medical images are given as input to the system.

2. For given input images, we apply the Sobel edge detection

3. For filtered images, color features are extracted using the color histogram.

4. We generate our index data base.

5. Finally, Searching and Retrieval process has been performed using one of the similarity measures such as Euclidean distance finding method.

The following [Figure2] depicts the architecture of our CBIR system:

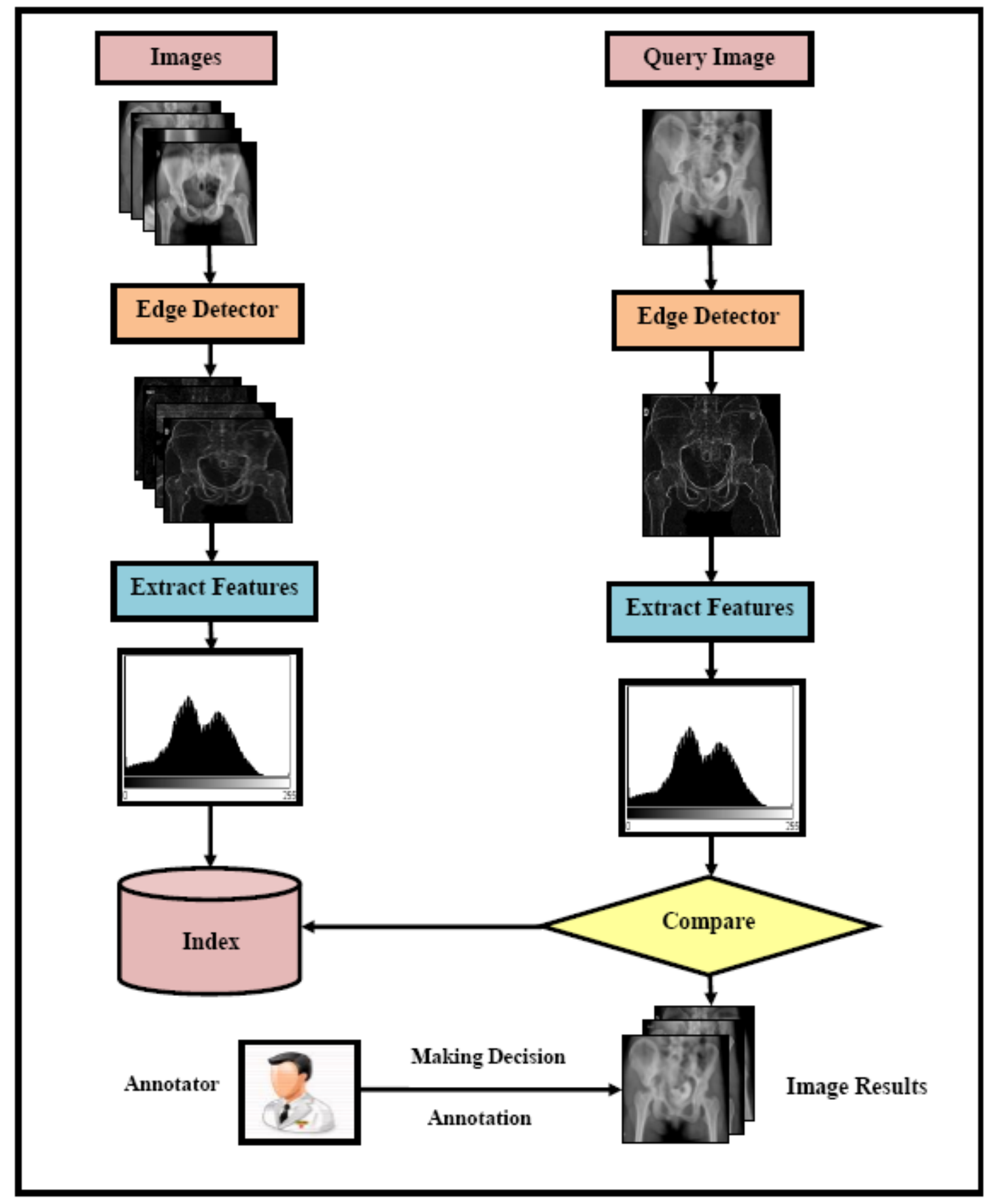

Figure 2. Architecture of our CBIR system 


\subsection{Edge Detection Algorithm}

Edge detection is one of the fundamental steps in image processing and image analysis techniques. The need of edge detection is to find the discontinuities in depth, discontinuities in surface orientation, changes in material properties and variations in scene illumination. Various experiments proved that the Sobel edge detection algorithm is fast and efficient in identifying the edges. However while taking into consideration both visual results and speed, Sobel [32] is identified as a suitable algorithm for detecting edges from the x-ray images. The Sobel method is identified as the fastest algorithm and the efficient in identifying the edges clearly in the same time. The following [Figure 3(a) and 3(b)] depicts the results of before and after edge detection.

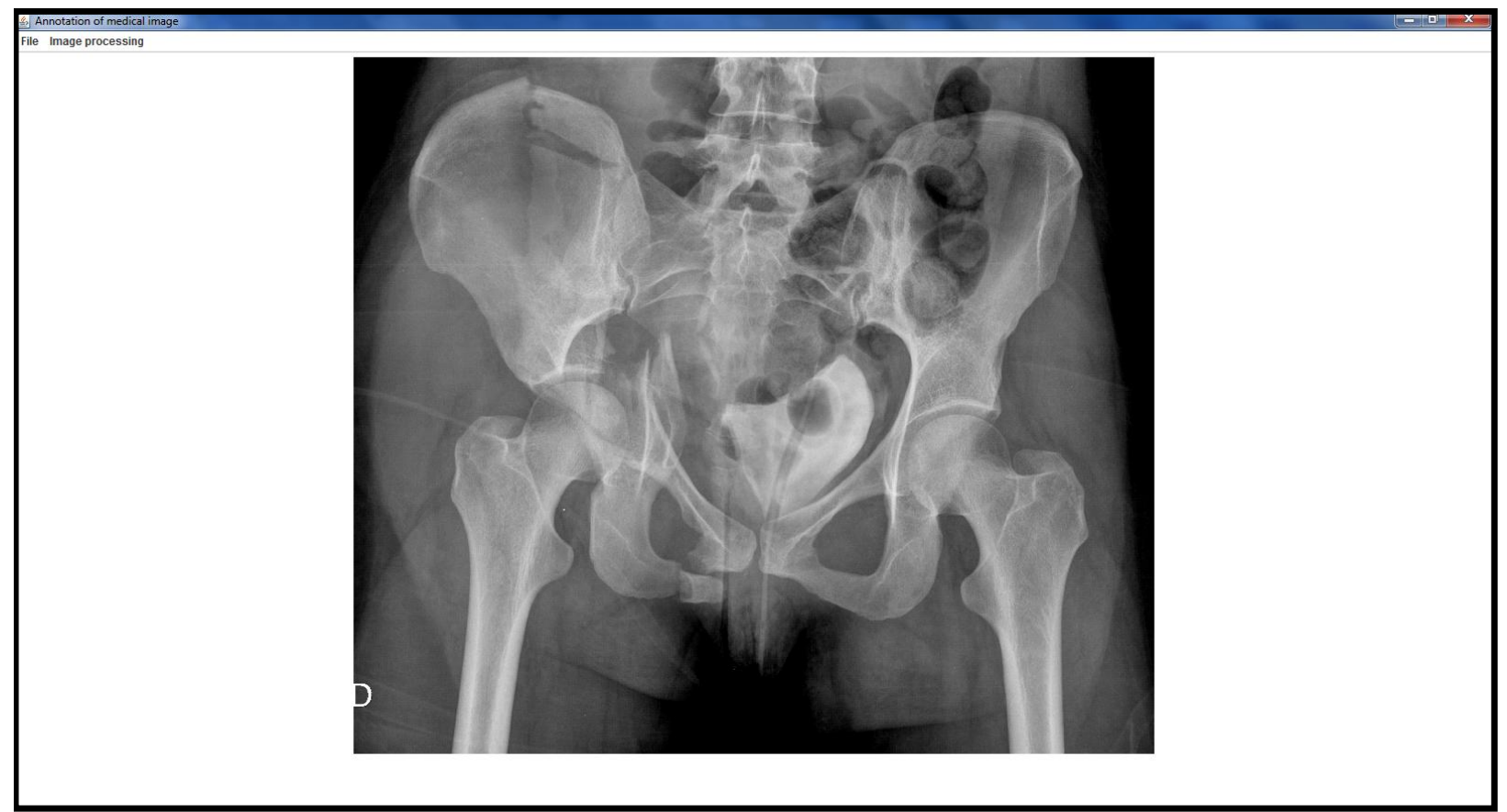

Figure 3 (a). The original image

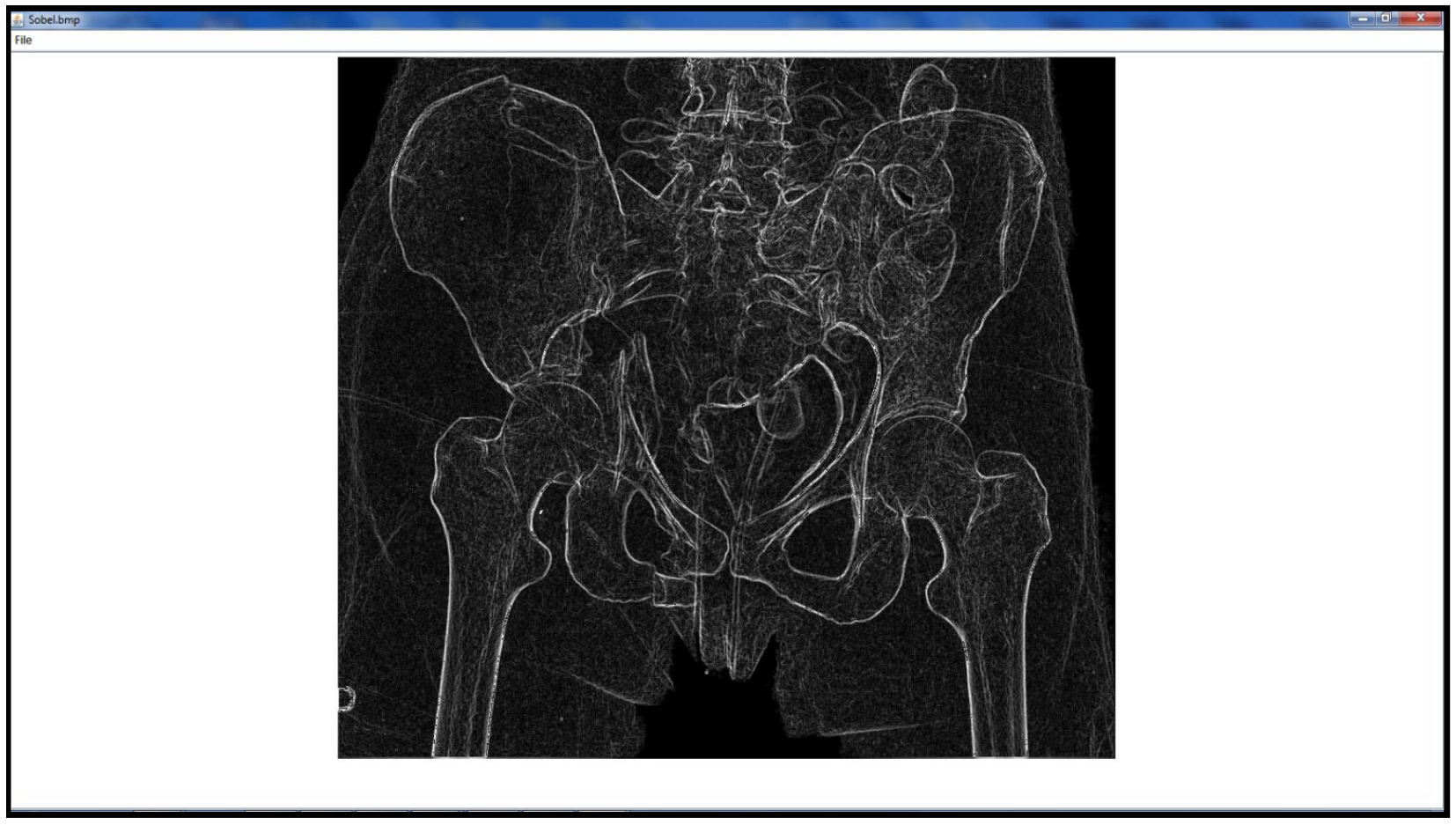

Figure 3 (b). Sobel Edge detector 


\subsection{Feature Extraction}

The precision of image indexing and image retrieval mainly based on image feature extraction. More distinguished image features will yields better results in indexing and retrieval process. In this work, we have extracted color features are extracted using the color histogram technique [33]. The color feature is one of the most widely used visual features in image retrieval. Because the human vision system is more sensitive to color information than grey values of images [34]. The color histogram describes the different colors distribution in an image in a simple and computationally efficient manner. The following [Figure 4] depicts the results of feature extraction.

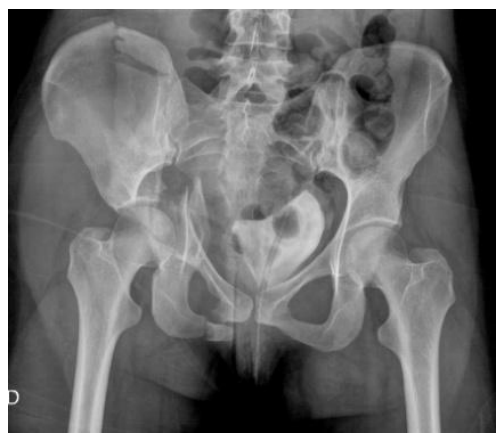

Original Image

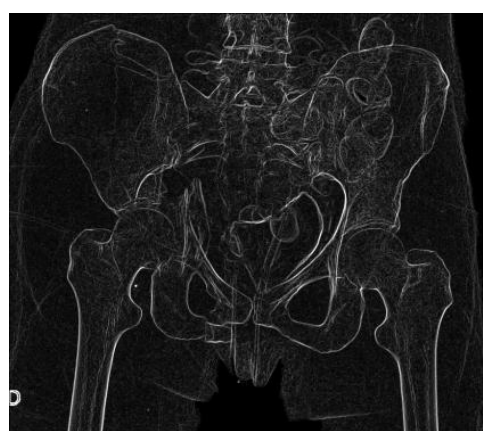

Sobel Edge Detector

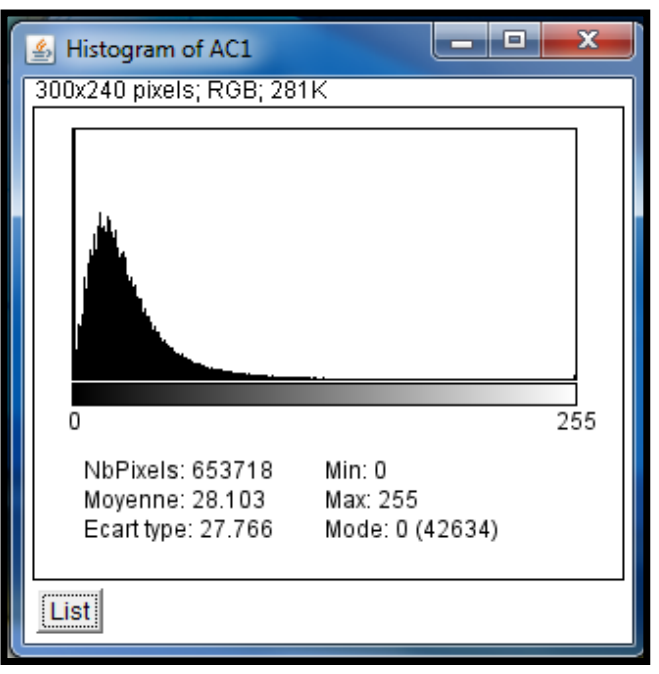

Color Histogram

Figure 4. Feature Extraction

\subsection{Image Indexing}

Content-based image retrieval (CBIR) aims at searching image databases for specific images that are similar to a given query image based on matching of features derived from the image content. In this work, the color features are extracted using the color histogram. In our case we work with the histogram for the gray level of each image. The histogram gives the number of pixels for each color: the histogram gives the number of pixels from level 0 until level 255. Then, these features are used for retrieval process.

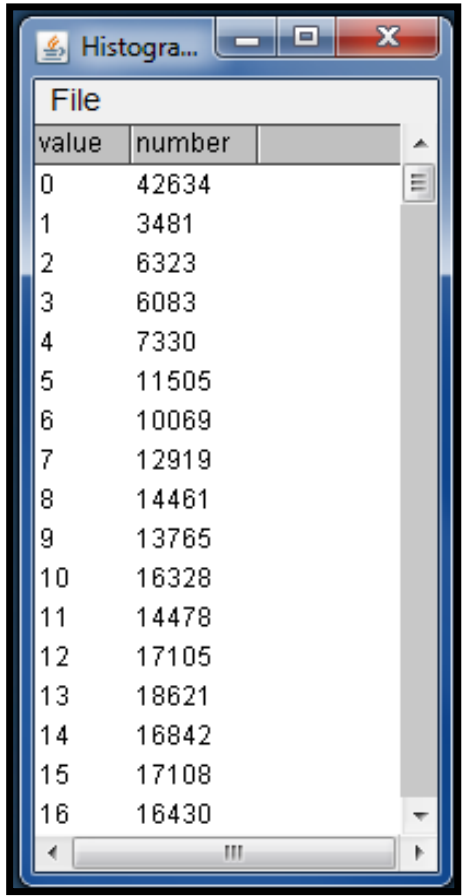

Figure 5. Image Indexing 


\subsection{Image Searching and Retrieval}

There are various image retrieval methods that have been applied in various applications. One among which is Euclidean distance method for image similarity measurement. Euclidean distance gives the best results for image searching [14]. In this work, we have used Euclidean distance method to retrieval of similar images from the database. When query image is given, the color feature of that image is identified and which is compared with values, from the data base of index, one by one by using Euclidean distance method. By comparing query image with others images in data base, the distance between images will be finding and shortest distance will be considered as best matching image in that matching process. Similarly, one after one best matching images will be finding by measuring the one after one shortest distance in to retrieve the similar images from the database. The distance $\mathrm{d}$ between two image calculated by using the following equation.

$$
d=\sqrt{ } \sum_{i=0}^{n}\left(h_{q}(i)-h_{d}(i)\right)^{2}
$$

Where $h_{q}$ is the $i^{t h}$ color feature of the query image, and $h_{d}$ is the $i^{\text {th }}$ color feature of the data base image. The following [Figure 6] depicts retrieval results.

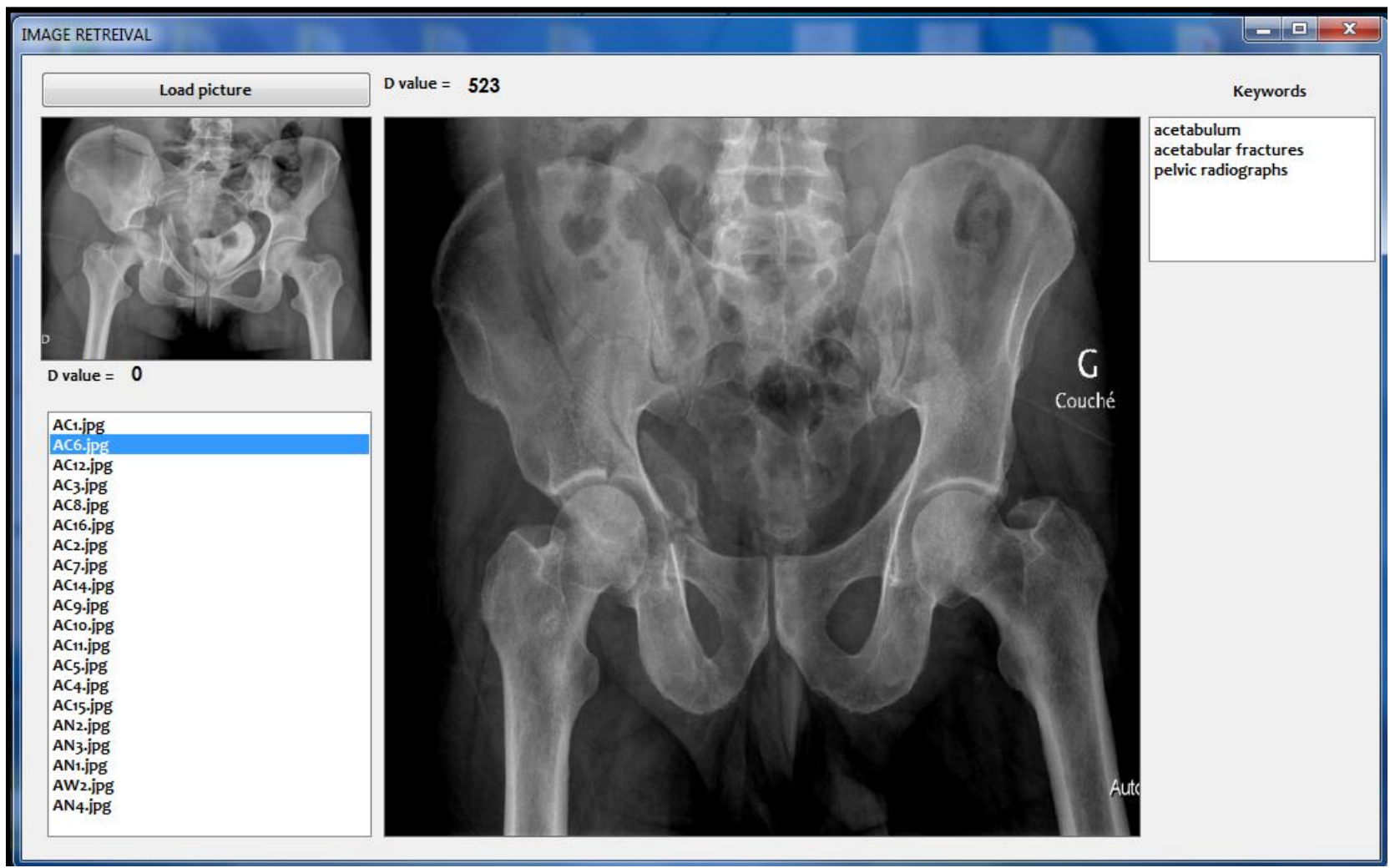

Figure 6. Retrieval Results

\subsection{Annotation}

From the list of image results, the annotator finds the most similar image to the query image. He will annotate every image with the keywords associated to the image retuned by the system. The similarity is made by a round of the database annotation whose specialty fixed in advance by the annotator and the class of the disease with the source image. The annotator selects keywords and uses them to annotate the new image. Figure 7 shows some similar medical images 

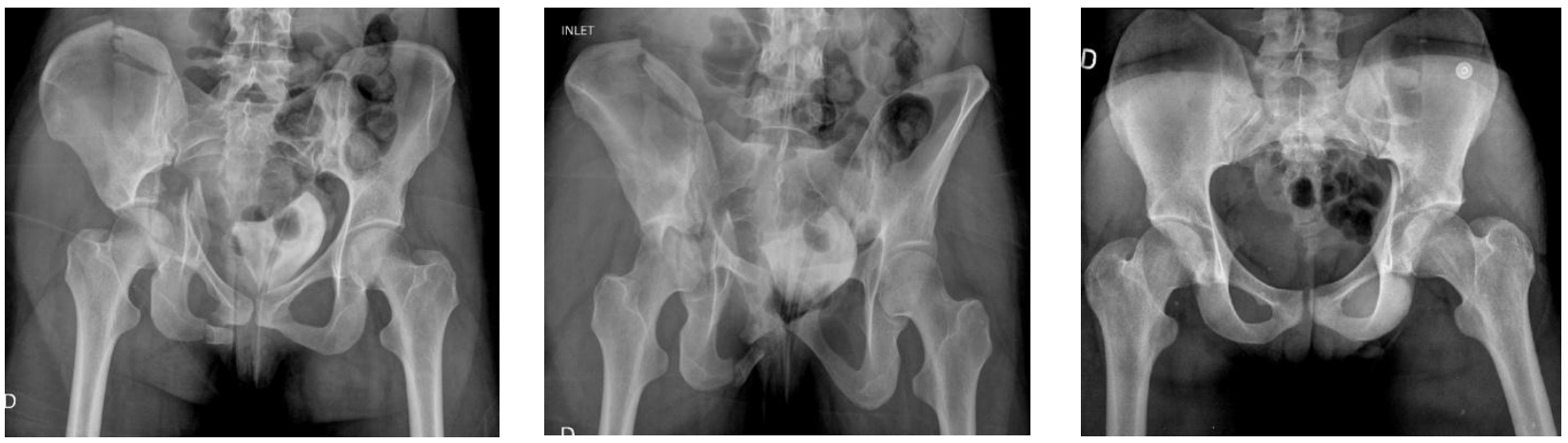

Figure 7. Example of similar medical images

\section{EXPERIMENTAL AND RESULT ANALYSIS}

We used java in the development of our system and for and the data base used was MySQL. We used with imagej API for the implementation of our work. We use the database Iconocerf is the digital image bank of Radiology of the Council of France (CERF) and The French Society of Radiology (SFR). This database contains 13,715 images of 3860 with clinical cases.

\subsection{Retrieval performance of the system}

We used the data base with 11 tests initially for testing the retrieval performance of the system. To measure this, precision and recall parameters were used and it was measured by using the following relationships:

$$
P=r / n
$$

Where, r-number of relevant images, and, n-number of retrieved images

$$
R=r / m
$$

Where, r-number of relevant images, and, m-total number of relevant images in data base.

The following [Table1] provides the precision and recall results recorded for various test images using our system.

TABLE I. PRecision AND ReCALl RESUlts RECORDED FOR VARIOUS TEST IMAGES

\begin{tabular}{|c|c|c|c|c|c|c|}
\cline { 2 - 7 } \multicolumn{1}{c|}{} & $\mathbf{1}$ & $\mathbf{2}$ & $\mathbf{3}$ & $\mathbf{4}$ & $\mathbf{5}$ & $\mathbf{6}$ \\
\hline Recall (\%) & 0 & 10 & 20 & 30 & 40 & 50 \\
\hline Precision (\%) & 100 & 85 & 75 & 57 & 55 & 49 \\
\hline
\end{tabular}

\begin{tabular}{|c|c|c|c|c|c|}
\cline { 2 - 6 } \multicolumn{1}{c|}{} & $\mathbf{7}$ & $\mathbf{8}$ & $\mathbf{9}$ & $\mathbf{1 0}$ & $\mathbf{1 1}$ \\
\hline Recall (\%) & 60 & 70 & 80 & 90 & 100 \\
\hline Precision (\%) & 35 & 25 & 19 & 11 & 2 \\
\hline
\end{tabular}

We will evaluate the performance of the proposed method with other systems which summarizes the obtained values for each MAP testing. We note that our approach works well on searching for similar images compared to other systems: the first is a CBIR system based directly on the application of color image to the image directly; the second is a CBIR system based on the image measures [14]. The following [Table2] shows the difference between the three approaches. 
TABLE II. AVERAGE PRECISION RESUltS

\begin{tabular}{|c|c|}
\hline CBIR System & MAP \\
\hline Our system & 0.466 \\
\hline Histogram system & 0.355 \\
\hline Measures system & 0.300 \\
\hline
\end{tabular}

These global observations are confirmed by the curves of precision / recall shown in Figure 8. Figure 8 shows the difference in behavior between the three approaches.

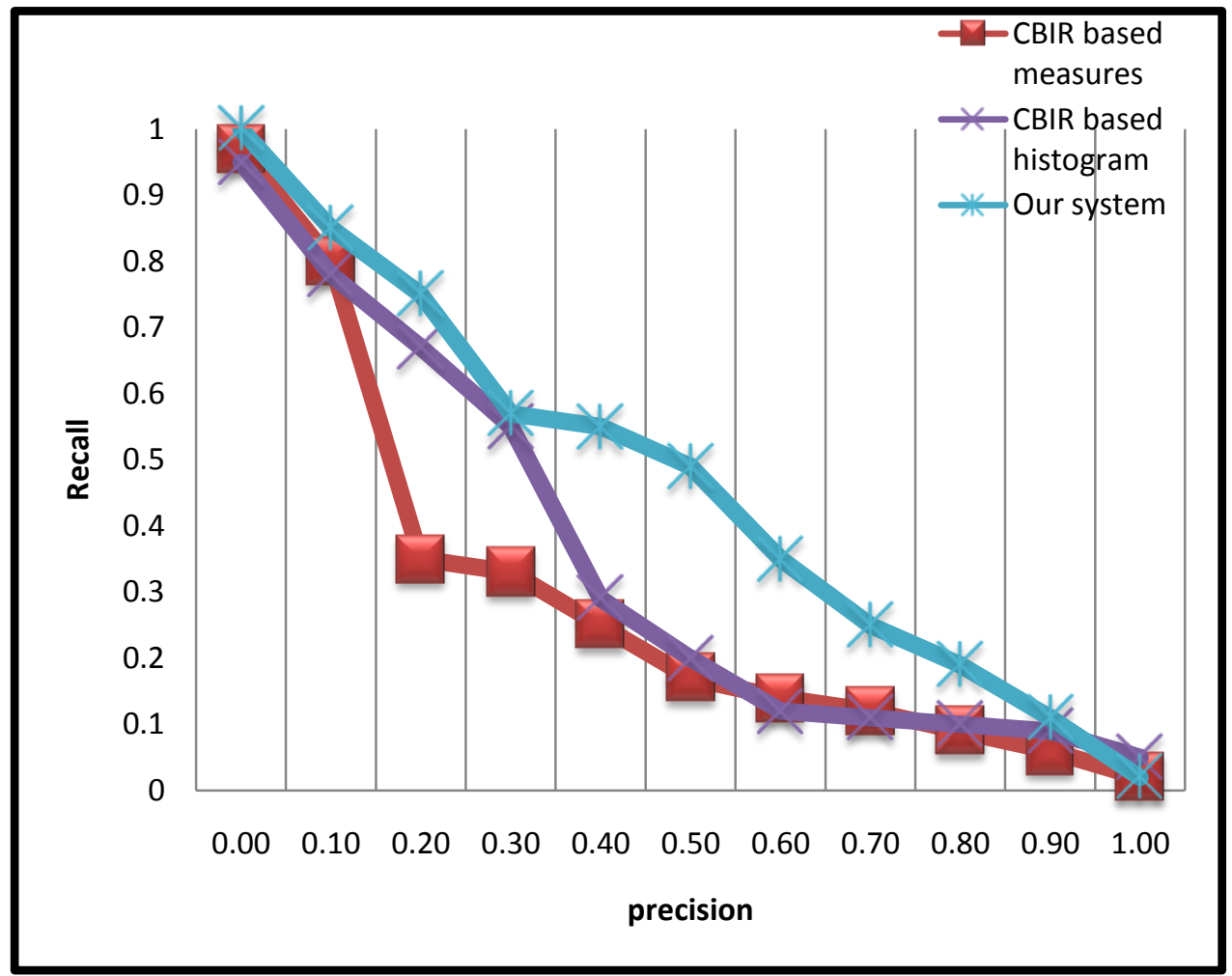

Figure 8. Courbe precision / recall for different Methods of radiological images retrieval

\subsection{Interest points}

In this part, we will introduce the effect of our idea by changing the extracted feature, from color features to finding interest points for an image. Interest points are themselves the result of an operation comprehending the full spatial domain $(X, Y)$. We went to use SURF algorithm. The SURF is an invariant interest point descriptor for finding correspondences between two images of the same scene or objects. The motivation for the development of the SURF is to speed up this generic correspondence process. The increase of number of interest points is very important for direct image matching, and to find the same image in a collection. We used the imagej for testing the idea. 


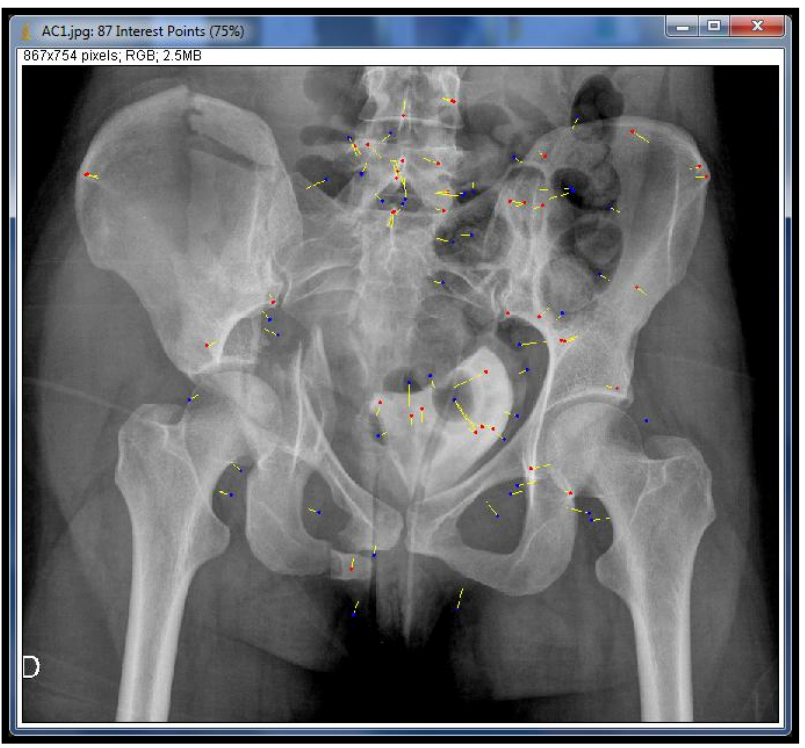

The interest points directly from the original image

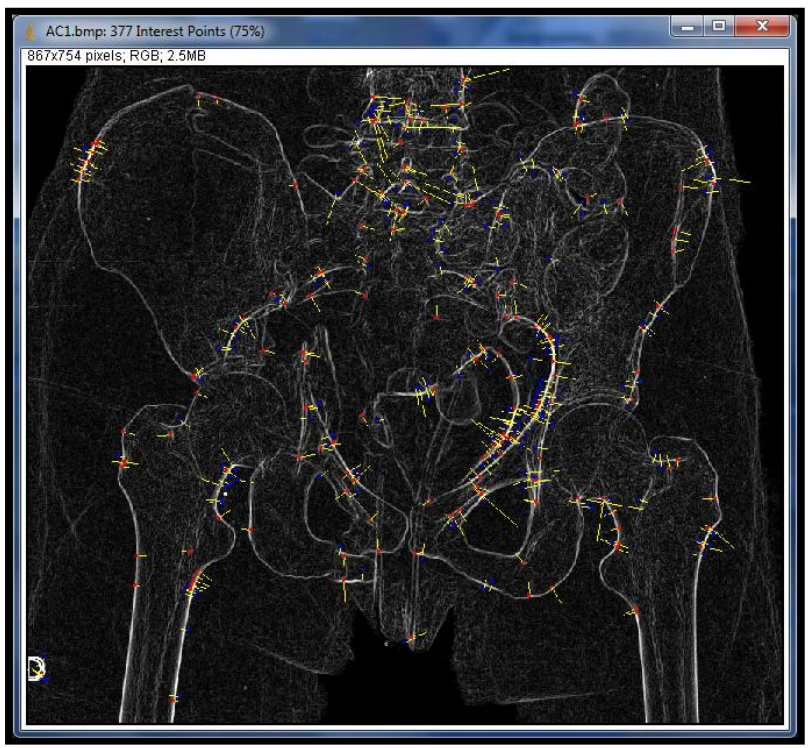

The interest points using our approach

The number of interest points increase from 87 to 377 interest points, which, it is prove the good effect for our approach in the improvement of image retrieval. The CBIR based edge detection gives more accurate results compared to results of direct work with image.

\section{CONCLUSION}

In this paper, we described our approach about new Image annotation based a new CBIR approach for the medical images. We have used Sobel edge detection algorithm for extracting the edge and we have used the color histogram for extracting the color features from the processed image. After extracting the color feature, which are given as input for indexing the images. Then, we used the indexed image for making easy for applying retrieval algorithm in order to retrieve the relevant images from the database. This work retrieves the images from the huge medical database as required by the doctors for their further evaluation about their patients' diseases. So that doctors can give proper treatment to their patients in proper time.

Our future work is to provide symptoms description, remedies for the diseases along with medical images. As perspectives, we plan to the improvement of our system by using other image descriptor and making fusion between them for having better results.

\section{REFERENCES}

[1] L. K. Barnard, P. Duygulu, D. Forsyth, N. Freitas, D. Blei, and M. Jordan, "Matching words and pictures", JMLR, 2003.

[2] A. Farhadi, I. Endres, D. Hoiem, and D. Forsyth, "Describing objects by their attributes" CVPR, 2009.

[3] X. Wang, L. Zhang, M. Liu, Y. Li, and W. Ma," image search to annotation on billions of web photos" CVPR, 2010

[4] J. Weston, S. Bengio, and N. Usunier, "Large scale image annotation: Learning to rank with joint wordimage embeddings", Machine Learning Journal, 2010.

[5] Igor Francisco Areias Amaral, "Content-Based Image Retrieval for Medical Applications “, October, 2010.

[6] B. Saida., "Recherche d'images par contenu", 2007.

[7] T. Deselaers, D. Keysers, and H. Ney, "Flexible Image Retrieval Engine: Image CLEF 2004 Evaluation“. In
Advances in Multilingual and Multimodal Information Retrieval, 5th Workshop of the Cross-Language Evaluation Forum, CLEF'04, pages 688-698, 2004.

[8] H. Muller, W. Muller, S. Marchand-Maillet, S. March, T. Pun, and D. M. Squire. "Strategies for positive and negative relevance feedback in image retrieval", In The 15th International Conference on Pattern Recognition, ICPR'00, pages 1043-1046, 2000.

[9] W. D. Bidgood. "the SNOMED DICOM microglossary: controlled terminology resource for data interchange in biomedical imaging". DOLAP', 1998.

[10] S. J. Weston, S. Bengio, and N. Usunier, "Large scale image annotation: Learning to rank with joint wordimage embeddings", Machine Learning Journal, 2010.

[11] Stéphane Clinchant, Julien Ah-Pine, Gabriela Csurka," Semantic Combination of Textual and Visual Information in Multimedia Retrieval”, 2011

[12] K Yiannis Gkfous, Anna Morou and Theodore Kalamboukis, "Combining Textual and Visual Information for Image Retrieval in the Medical Domain", 2011

[13] Brodeur, J., Badard, B.: Modeling with ISO 191xx standard. In: Shekhar, S.; Xiong, H. (Eds.). Encyclopedia of GIS. Springer-Verlag, pp. 691--700, 2008.

[14] Swarnambiga Ayyachamy, and Vasuki S. Manivannan, "Distance Measures for Medical Image Retrieval". Vol. 23, pp.9-21, 2013.

[15] Garg, R., Mittal, B. and Garg, S. Histogram Equalization Techniques For Image Enhancement, International Journal of Electronics \& Communication Technology, Vol. 2, Issue 1, Pp. 107-111., 2011

[16] Donnelley, M. "Computer aided long-bone segmentation and fracture detection, a thesis presented to the Flinders University of South Australia in total fulfillment of the requirements for the degree of Doctor of Philosophy Adelaide", South Australia, Chapter 6, and P. 122. 2008. 
[17] Tamisiea D.F. Radiologic aspects of orthopedic diseases, Mercier LR, ed. Practical Orthopedics, 6th ed. Philadelphia, Pa: Mosby Elsevier; Chap 16, 2008.

[18] J. Matthews "An introduction to edge detection: The sobel edge detector" Available at http://www.generation5.org/content/2002/im01.asp, 2002

[19] Harshlata Vishwakarma1 and S.K.Katiyar," COMPARATIVE STUDY OF EDGE DETECTION ALGORITHMS ON THE REMOTE SENSING IMAGES USING MATLAB”, Vol. No. 2, Issue No. VI, December,2011

[20] Long L.R., Antani T. and Thoma G.R.,"CBIR in medicine: retrospective assessment state of the art and future directions ", Vol 4. No. 1, 2009.

[21] Xue Z., Long L.R., Antani T. and Thoma G.R, "A Web accessible content based cervicographic image retrieval system", in proceedings of SPIE Medical Imaging, 2008.

[22] Long L.R, Hsu W., and Antani S., "SPIRS: A framework for content based image retrieval from large Biomedical Batabases", in proceedings of MEDINFO, 2010 .

[23] Deserno T.M., Long L.R., Plodowski B., and Spitzer K. , "Extended query refinement for medical image retrieval".journal of digital imaging, 2007.

[24] Antani S, Güld M.O.,Long L.R., Antani T. and Thoma G.R, "Interfacting global and local CBIR systems for medical image retreival",2007

[25] Igor Francisco Areias Amaral, "CBIR for medical Applications". Porto, October 2010.

[26] A. Grace Selvarani and S.Annadurai, "Content Based Medical Image Retrieval System using Shape and Texture Features", ICGST-BIME Journal, Vol 8, Issue 1, December 2008
[27] Muller, H., N.Michoux, D.Bandon and A.Geissbuhler, "A review of content based image retrieval systems in medical applications-clinical benefits and future directions", International Journal of Medical Information., pp: 73:13, 2004

[28] Petrakis, Euripidies G.M and C.Faloutsos, "ImageMap: An Image indexing Method Based on Spatial Similarity", IEEE Trans on Knowledge and Data Eng., 14(5):979987, 2002.

[29] Chi-Ren Shyu, Carla E.Brodley, Avinash C.Kak, Akio Kosaka, "ASSERT: A Physician-in-the-Loop ContentBased Retrieval System for HRCT Image Databases", Computer Vision and Image Understanding,Vol.75,Nos.1/2,July/August,pp.111- 132, 1999.

[30] Chbeir, R., Y.Amghar, and A.Flory,"MIMS: A Prototype for Medical Image Retrieval", Proctor the 6th Conference on Content-Based Multimedia Information Access- Recherched'Informations Assist'ee par Ordinateur.Paris, France, 2000.

[31] Prof. K.Wanjale, Tejas Borawake and Shashideep Chaudhari, "Content Based Image Retrieval for Medical ImagesTechniques and Storage Methods-Review Paper", Volume 1- No.19, February 2010.

[32] S.K. Mahendran, "A Comparative Study on Edge Detection Algorithms for Computer Aided Fracture Detection Systems", Volume 2, Issue 5, November 2012

[33] .Swain, M.J. and D.H.Ballard, "Color Indexing", International Journal of Computer Vision, 7:11-32, 1991

[34] Lim, J.H., S.J.Jesse and Luo Suhuai, "A Structured Learning Approach to Semantic Photo Indexing and Query", Asia Information retrieval symposium,13-15 October 2005,Jeju Island, Korea. 